\title{
NEW SUPERLUMINAL QUASARS
}

\author{
T. J. Pearson, P. D. Barthel, A. C. S. Readhead, and C. R. Lawrence \\ Owens Valley Radio Observatory \\ California Institute of Technology \\ Pasadena, California 91125 \\ USA
}

Since 1977 we have been engaged on a project to survey the milliarcsecond structure and internal motions of a complete, unbiased sample of 65 radio sources (Pearson and Readhead 1984). One of the goals of the project was to discover more superluminal sources and find out how common they are. The sample contains three established superluminal sources (3C 179, 3C 345, and BL Lacertae); so far we have discovered three new superluminal quasars $(0850+581,1642+690$, and $1928+738)$, and a fourth $(3 \mathrm{C} \mathrm{216})$ that may be superluminal. Thus at least six out of the 65 sources are superluminal, and we expect to find more as our observations proceed.

The complete sample was selected from the NRAO-MPIfR 5-GHz Strong Source Surveys S4 and S5 by the following criteria: (a) declination (1950) $>35^{\circ}$, for good $u, v$ coverage with existing telescopes; (b) galactic latitude $|b| \geq 10^{\circ}$; (c) total flux density at $5 \mathrm{GHz} \geq 1.3 \mathrm{Jy}$ (at the epoch of the original surveys). Twenty of the objects in the sample are classical double radio sources, while the remaining 45 are compact, core-dominated sources with $\gtrsim 95 \%$ of their flux density arising in a region smaller than about $1^{\prime \prime}$. We now have first-epoch VLBI maps of $\mathbf{4 1}$ of the core-dominated sources in the sample and second-epoch maps of 20.

We are in the process of obtaining high-quality optical spectra of the entire sample of 65 sources using the double spectrograph on the Palomar 200-inch telescope. These observations have already provided a number of new redshifts (Lawrence et al. 1986), including that of $1642+690$.

It is clear from the VLBI observations that not all compact radio sources are superluminal. In particular, there is a class of "compact double" sources that are identified with galaxies or empty fields, show little flux-density variation, and have low polarization $(<1 \%)$; none of these has shown large structural changes. On the other hand, at least half the sample consists of unresolved objects and asymmetric "core-jet" sources that are highly polarized and highly variable in both total flux density and structure. This class includes most of the quasars and BL Lac objects. The six known superluminal sources all have a "core-jet" structure, and it is quite possible that all of the objects of this type will turn out to be superluminal when they have been studied sufficiently well.

$0850+581, z=1.322$ : We have maps of $0850+581$ at only one frequency, but they show an asymmetric structure that can be described as a "core-jet," with the mil- 
liarcsecond jet aligned with a curved, large-scale jet, $10^{\prime \prime}$ long. The change in the structure is very suggestive of superluminal motion along the jet, with $v / c=(4.6 \pm 2.3) h^{-1}$, and further observations are in progress to confirm the result.

$0906+430$ (3C 216), $z=0.669: 3 \mathrm{C} 216$ is unlike the other quasars presented here in that the VLBI observations show a milliarcsecond jet aligned roughly perpendicular to the major axis of the large-scale emission; the only comparable source is the quasar 3C 309.1. Both 3C 216 and 3C 309.1 are steep-spectrum compact sources. The change in structure is ambiguous: it could be due to a weak component in the "jet" having brightened in the 4.5-yr period between the two observations; but a plausible explanation is that the change is due to the motion of a component along the jet, with $v / c=(11.5 \pm 2.0) h^{-1}$.

1642+690, $z=0.751$ (Pearson et al. 1986): The radio source $1642+690$ has a dominant, compact core with a jet extending $5^{\prime \prime}$ to the south. The VLBI maps of the core show that the milliarcsecond structure is similar to that of the superluminal sources 3C 273 and 3C 345. A milliarcsecond jet is closely aligned with the arcsecond jet, and a "knot" in the jet is moving outwards along the jet at $0.34 \mathrm{mas} / \mathrm{yr}$, an apparent transverse velocity of $v / c=9.3 h^{-1}$.

1928+738, $z=0.302$ (Eckart et al. 1985): The VLBI maps of $1928+738$ show a self-absorbed "core" with spectral index $\alpha \approx 0.0$ and a long "jet" extending 17 mas from the core in position angle $165^{\circ}\left(50 h^{-1} \mathrm{pc}\right)$. The jet has a spectral index $\alpha \approx-0.5$ and contains at least nine components, five of which were detected at both epochs. The most plausible alignment of the maps from the two epochs indicates that all five of these components have a proper motion relative to the core of $\sim 0.6 \mathrm{mas} /$ year, or apparent transverse velocities $v / c \sim 7.5 h^{-1}$.

\section{REFERENCES}

Eckart, A., Witzel, A., Biermann, P., Pearson, T. J., Readhead, A. C. S., and Johnston, K. J.: 1985, Astrophys. J. Letters 296, L23-L26.

Lawrence, C. R., Pearson, T. J., Readhead, A. C. S., and Unwin, S. C.: 1986, Astron. J. 91, in press.

Pearson, T. J., and Readhead, A. C. S.: 1984, IAU Symp. 110, 15-24.

Pearson, T. J., Barthel, P. D., Lawrence, C. R., and Readhead, A. C. S.: 1986, Astrophys. J. Letters 300, L25-L29. 